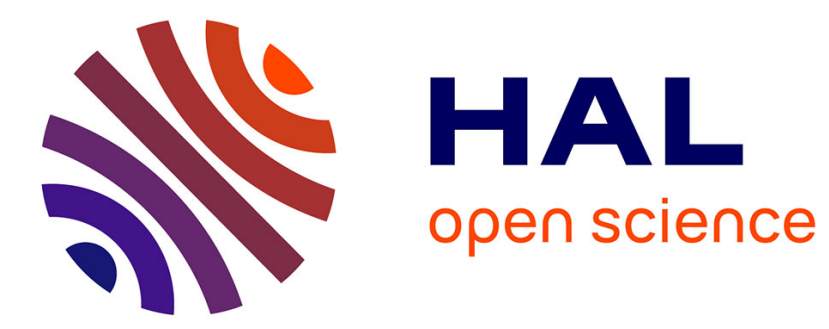

\title{
Grégoire le Grand, Alcuin et l'idéologie carolingienne
}

Bruno Judic

\section{To cite this version:}

Bruno Judic. Grégoire le Grand, Alcuin et l'idéologie carolingienne. Wojciech Falkowski; Yves Sassier. Le monde carolingien: Bilan, perspectives, champs de recherches, Brepols, 2009, 978-2-503-53202-8. hal-01550551

\section{HAL Id: hal-01550551 \\ https://hal-univ-tours.archives-ouvertes.fr/hal-01550551}

Submitted on 29 Jun 2017

HAL is a multi-disciplinary open access archive for the deposit and dissemination of scientific research documents, whether they are published or not. The documents may come from teaching and research institutions in France or abroad, or from public or private research centers.
L'archive ouverte pluridisciplinaire HAL, est destinée au dépôt et à la diffusion de documents scientifiques de niveau recherche, publiés ou non, émanant des établissements d'enseignement et de recherche français ou étrangers, des laboratoires publics ou privés. 
Dans Le monde carolingien: Bilan, perspectives, champs de recherches. Actes du colloque international de Poitiers, Centre d'Etudes supérieures de Civilisation médiévale (18-20 novembre 2004), édités par Wojciech FALKOWSKI et Yves SASSIER Turnhout, Brepols 2009, p. 105-120.

Bruno JUDIC

Grégoire le Grand, Alcuin et l'idéologie carolingienne

Les synodes anglais de 786 sont connus grâce à un rapport de Georges d'Ostie adressé au pape Hadrien Ier. Sous la forme d'une lettre, il s'agit en réalité des actes de ces synodes ou plus exactement de la même liste de décisions ratifiées à la fois en Northumbrie et en Mercie. Ces décisions synodales sont souvent présentées avec beaucoup d'arguments et offrent ainsi une grande richesse d'informations. On s'est intéressé particulièrement à la place de ce concile dans les conciles anglais du haut moyen âge ainsi qu'au rôle possible joué par Alcuin dans la rédaction ou dans l'inspiration de ces décisions (1). Je m'attacherai à retrouver dans ce texte tout ce qui peut relever d'une influence de Grégoire le Grand, directe ou indirecte, et 
surtout qu'elle vienne de Rome (par Georges d'Ostie) ou d'Angleterre même (par Alcuin).

Il convient d'abord de rappeler rapidement le contenu de ces synodes. Le rapport de Georges d'Ostie comprend vingt canons (2). Les dix premiers concernent spécifiquement le clergé. Le canon 1 insiste sur la doctrine du concile de Nicée, sur la nécessité de réunir des assemblées synodales et sur le rôle du clergé pour prêcher la foi telle qu'elle est définie dans les six conciles universels. Le canon 2 concerne le baptême; il doit être associé à la connaissance du symbole et de l'oraison du Seigneur et au rejet de Satan. Le canon 3 demande aux évêques de visiter leurs diocèses, de prêcher et de réprimer les pratiques païennes; ce canon est particulièrement long; il est largement composé de citations scripturaires pour définir idéalement la figure de l'évêque. Le canon 4 demande une distinction nette entre la vie canoniale et la vie monastique; dans les deux cas il faut rejeter les vêtements luxueux; il faut fréquemment lire les canons des six conciles universels. Le canon 5 concerne la succession d'un abbé ou d'une abbesse après leur décès; il faut trouver un homme convenable dans la communauté ou le prendre dans un autre monastère. Le canon 6 concerne l'ordination d'hommes éprouvés qui doivent rester attachés à leur église sauf autorisation. Le canon 7 concerne le respect des heures canoniques. Le canon 8 concerne le respect des privilèges accordés aux églises par le siège romain. Le canon 9 concerne le respect du jeûne par les clercs. Le canon 10 concerne différentes prescriptions pratiques pour la célébration du culte: le prêtre ne doit pas être jambes nues, le pain doit être du pain et pas de la croûte, le calice ne doit pas être en corne.

Les dix canons suivants concernent les laïcs. Le canon 11 concerne les rois et les princes qui doivent mener leur gouvernement avec prudence et discipline. Mais ils doivent aussi obéir aux évêques qui rendront compte de leur conduite auprès de Dieu. C'est un canon 
très long, largement constitué de citations scripturaires. Le canon 12 concerne les conditions d'accès à la royauté: les rois ne doivent pas être choisis par des hommes mauvais mais par les prêtres et les anciens du peuple. Un roi ne peut être issu d'un adultère ou d'un inceste mais seulement d'un mariage légitime. Personne n'a le droit de tuer le roi car il est un oint du Seigneur (christus Domini). Un prêtre ou un évêque qui aurait pris part à un tel meurtre sera destitué. Ce canon est également assez long car il est aussi fondé sur plusieurs citations scripturaires. Le canon 13 concerne les puissants et les riches. Ils doivent juger avec justice. Ce canon aussi est nourri de citations scripturaires. Le canon 14 interdit la fraude et la violence et rappelle que les églises ne doivent pas être imposées de tributs injustes. D'ailleurs les rois et les évêques doivent observer la concorde. Le canon 15 interdit les mariages incestueux, avec des parents proches et consanguins; il interdit le mariage avec l'épouse d'autrui et avec une servante de Dieu. Le canon 16 interdit l'héritage légitime aux bâtards et aux enfants de prostituées. Il interdit également le mariage avec une vierge vouée à Dieu. Il exige le mariage légitime pour fuir la fornication. Le canon 17 exige le versement de la dîme avec plusieurs arguments scripturaires; il interdit l'usure; il exige enfin la régularité des poids et mesures. Le canon 18 exige le respect des vœux avec plusieurs arguments scripturaires qui en font un canon assez long. Le canon 19 rejette les rites des païens et condamne les tatouages. Il interdit les vêtements des païens ainsi que les coutumes d'incision sur les naseaux, les oreilles et la queue des chevaux. Il interdit les sorts et il interdit de manger la viande de cheval. Le canon 20 rappelle la nécessité de l'eucharistie et de la pénitence. Viennent ensuite les souscriptions distinguées en deux groupes. D'abord le groupe des souscriptions correspondant au synode réuni en Northumbrie; on y trouve l'archevêque de York Eanbald, le roi de Northumbrie Aelfwald, cinq évêques, trois grands laïcs et deux abbés 
(3). Ensuite le rapport évoque le départ de la délégation romaine vers la Mercie en compagnie de deux lectores délégués par le roi et l'archevêque, Alcuin et Pyttel. On trouve ainsi naturellement à la suite le deuxième groupe de souscription avec l'archevêque de Canterbury Iaenberth, le roi de Mercie Offa, douze évêques, quatre abbés et quatre grands laics (4).

Il faut d'abord souligner la référence explicite à Grégoire le Grand dans le préambule de la lettre. Les légats romains allaient retirer la mauvaise herbe qui pouvait étouffer "la semence que le bienheureux pape Grégoire avait semé par la bouche de saint Augustin”. Cette référence explicite à Grégoire le Grand n'a rien d'étonnant dans un texte concernant l'Angleterre. Grégoire est en effet l'apôtre des Anglais pour Bède le Vénérable et, à Rome, dès le VIIè siècle, on associe le nom de Grégoire à l'Eglise anglaise. Plus près de 786, un précédent synode anglais, en 747, à Cloveshoe, avait insisté sur le culte de saint Grégoire mis en relief dans le canon 17. Ce culte est en réalité bien plus ancien puisque ce canon mentionne aussi le culte de saint Augustin de Canterbury qu'il s'agissait ainsi de promouvoir en s’appuyant sur la ferveur dont bénéficiait déjà saint Grégoire. Le synode de Cloveshoe faisait aussi référence à Grégoire à la fin de son préambule: les évêques sont invités à échanger entre eux des paroles d'exhortation mutuelle à propos de leur fonction; ils trouveront un miroir splendide de leur fonction dans les homélies du bienheureux pape Grégoire et dans les décrets canoniques des saints pères (5). Ainsi Grégoire n'est pas seulement l'objet d'un culte fervent; il est l'auteur d'homélies qui doivent inspirer la vie religieuse et la vie pastorale. Bien sûr le rôle de Bède et de la "renaissance northumbrienne" est capital dans la diffusion des œuvres de Grégoire mais toute l'Angleterre est concernée. Boniface, originaire du sud de l'Angleterre, avait en effet fortement inspiré certaines décisions de Cloveshoe. Mais Grégoire est un pape 
romain et les légats de 786 ne peuvent pas l'ignorer. A cette date, Grégoire le Grand est désormais une figure éminente de la papauté elle-même. Le pape Hadrien Ier, le destinataire de la lettre de Georges d'Ostie, utilise les œuvres de Grégoire le Grand. Il y trouve des sources fondamentales de son action pontificale (6). Aussi faut-il lire avec attention dans cette lettre-rapport le récit de voyage.

Les légats sont partis de Rome, ils ont traversé le monde franc où ils reçoivent le soutien de Charlemagne qui leur adjoint son propre envoyé Wigbod. Ils parviennent enfin en Angleterre: "nous avons pris la route, mais celui qui tente par un vent contraire nous a mis des obstacles, et celui qui calme les flots (Ps. 88, 10) nous a transportés jusqu'au port du salut et malgré des périls nombreux il nous a poussés affligés et cependant intacts vers les rivages des Anglais". La traversée de la Manche a donc été difficile. Ces périls de la navigation rappellent l'angoisse qui saisit le groupe de moines romains envoyés par Grégoire en 596. Ils s'arrêtèrent en Provence, effrayés par le récit qu'on leur faisait de ce lointain pays barbare (7). Cela rappelle aussi la mort de l'abbé Pierre de Canterbury à Ambleteuse au nord de Boulogne au retour du concile de Paris de 614. Mais Georges d'Ostie exprime cette difficulté du voyage dans des termes empruntés à l'Enéide. On notera aussi que le "port du salut" est évidemment un thème très commun de la littérature spirituelle mais il a un arrière-plan grégorien. Les images maritimes du port (portus monasterii), du naufrage, des flots qui arrachent un navire mal amarré, de la tempête qui se déchaîne même dans la baie la mieux abritée, se trouvent dans la lettre de dédicace des Moralia in Job envoyée à Léandre de Séville. On les trouve aussi dans un des toutes premières lettres du pontificat de Grégoire, en octobre 590, adressée à Jean de Constantinople: "tout indigne et malade que je suis, j'ai reçu ce vieux navire tout brisé, qui fait eau de toute part; et dans la grosse tempête qui le secoue chaque jour ses 
planches pourries ont des craquements de naufrage" (ep. 1, 4). Heureusement pour les légats de 786, leur voyage s'est bien passé et la lettre nous rapporte aussi au moins implicitement leur itinéraire à l'intérieur même de l'Angleterre. Ils sont arrivés d'abord à Canterbury, puis ils ont rejoint la cour d'Offa roi de Mercie, sans précision (peut-être à Chelsea un peu en amont de Londres sur la Tamise). Là ils se sont séparés, Théophylacte de Todi est parti chez le roi des Merciens et vers la Bretagne (Britannie partes) — on doit donc supposer qu'il est parti vers l'ouest, la Bretagne désignant les royaumes bretons de l'actuel Pays de Galles Georges d'Ostie a continué vers le nord pour rejoindre la Northumbrie où l'archevêque de York dut envoyer un messager au roi qui se trouvait dans les régions boréales — donc encore plus loin au nord. Peut-être le roi se trouvait-il en Bernicie et est-il revenu vers le sud pour diriger le concile qui aurait eu lieu à Corbridge, sur le mur d'Hadrien (8). Après la fin de ce concile northumbrien, le légat a repris la route, comme l'indique le dernier paragraphe de la lettre, accompagné d'Alcuin et Pyttel, lectores, envoyés par le roi et l'archevêque. Ils rejoignent à nouveau le roi des Merciens dans le synode des Merciens (à Chelsea?) où l'on fait lire les canons du concile à la fois en latin et en theodisca (tam latine quam theodisce) (9). Les légats rappellent qu'aucun prêtre romain depuis saint Augustin de Canterbury n'est venu en Angleterre (10).

Pour justifier cet itinéraire à l'intérieur de l'Angleterre, Georges d'Ostie évoque la géographie: "parce que cet angle est étendu en longueur et en largeur". L'emploi du mot angulus pour désigner un pays est connu en latin classique et sert éventuellement à opposer un "petit" pays à l'ensemble du vaste monde. Cet emploi se retrouve chez saint Augustin (d'Hippone): alors un seul angle de la terre opposé à l'ensemble du monde fait référence à la Judée (11). Mais ici le mot angulus rapproché du peuple des Anglais (gens Anglorum), des 
rivages des Anglais (Anglorum orae) sonne comme un jeu de mots. Or ce jeu de mots se trouve précisément chez Grégoire le Grand. On connaît habituellement le jeu de mots sur Angli / angeli (qui n'est peut-être pas absent non plus de la lettre, cf. c. 11, mais c'est moins net); jeu de mots qui apparaît dans la Vita Gregorii de Whitby au tout début du VIIIè siècle puis chez Bède (H.E. II, 1). Chez Grégoire lui-même nous trouvons le jeu de mots sur angulus; il s'agit d'une lettre de juillet 598 au patriarche d'Alexandrie Euloge, Grégoire évoque la gens Anglorum in mundi angulo posita, il remercie son confrère pour ses prières et se réjouit de la conversion de dix mille Anglais qui ont été baptisés (ep. 8, 29). Grégoire avait évidemment les qualités de style pour manier la langue et un certain humour. Il n'est pas du tout impossible que le jeu de mots sur les anges vienne de lui. On se demandera quand même comment cette lettre à un patriarche d'Alexandrie pouvait être connue. Mais précisément à Rome, au temps d'Hadrien Ier, le registre des lettres de Grégoire est l'objet d'explorations empressées. Déjà Boniface en avait obtenu des copies. (Alcuin pourtant manifestera un peu plus tard son ignorance de certaines lettres). A cette date, le jeu de mots peut très bien faire partie des "bagages" du légat romain mais il pouvait aussi se trouver dans la tradition anglaise locale, tradition écrite comme le rappelle Alan Thacker à l'arrière-plan de la Vita de Whitby. On évoquera un dernier écho possible de cet “angle". Le livre de l'Exode évoque les quatre angles de l'Arche, qui sont les quatre parties du monde dans la Règle Pastorale de Grégoire le Grand repris par Bède dans le De tabernaculo. Les quatres angles de la terre sont aussi dans 1'Apocalypse et la conversion des Anglais est présentée par Grégoire au roi Ethelbert du Kent comme un signe vers la fin prochaine du monde.

L'influence de la Règle pastorale est en tout cas visible sur le canon 3 concernant les 
évêques. Il s'agit de tenir des synodes et de visiter le diocèse; il s'agit de prêcher aux fidèles. Mais ce canon développe toute une série de citations scripturaires sur la charge du pasteur. Catherine Cubitt a déjà signalé la reprise d'extraits des Sentences d'Isidore de Séville mais à l'arrière-plan des Sentences on peut y voir aussi l'influence de Grégoire le Grand. En effet presque toutes les citations scripturaires de ce canon se retrouve dans la Règle pastorale (12): I Tim 4, 1-2, “...proclame la Parole, insiste à temps et à contre-temps...” conclut le chap. 2, 4 sur l'urgence de la prédication. Is. 40, 9, "Monte sur une haute montagne, toi qui évangélises Syon" ouvre le chap. 2, 3 pour inciter le prédicateur à montrer le chemin au troupeau. Le canon 3 associe ensuite l'appel d'Isaïe à parler et l'encouragement à Jérémie comme dans le chap. 1, 7 où Isaïe s'offre spontanément tandis que Jérémie avoue son incapacité à parler. Ez. 13, 5, “Vous n'êtes pas montés contre l'ennemi et vous n'avez pas opposé un mur, pour tenir ferme dans le combat au jour du Seigneur" se retrouve dans le chap. 2, 4 à la suite d'un développement sur les mêmes thèmes, le pasteur ne doit pas craindre de dire librement où est le bien, il ne doit pas être comme un mercenaire qui fuit quand vient le loup, sinon il recevra ce reproche du prophète. La citation de Jean 10, 12, "le bon pasteur donne son âme pour ses brebis" est également dans le même passage du Pastoral. Enfin Mt 25, 21 sur le serviteur bon et fidèle évoque la parabole des talents, ce que fait aussi le chap. 3, 25: il s'agit de ceux qui pourraient prêcher mais ne le font pas par humilité excessive. C'est bien le même contexte de la nécessité de la prédication.

Dans le même groupe de canons concernant le clergé, on peut également regarder de plus près les canons 9 et 10 . Le canon 9 condamne le fait de prendre de la nourriture en secret sous-entendu quand on prétend jeûner. C'est même l'hypocrisie des Sarrasins (ou Saracènes), remarque évidemment liée au contexte du VIIIè siècle. Mais ensuite ce canon rapporte cette 
mauvaise pratique à des sépulcres blanchis par référence à Mt 23, 27, puis il cite Mt 6, 1 "faites attention à ne pas rendre votre justice devant les hommes, pour que vous soyez vus par eux..." Dans le chap. 3, 35 du Pastoral il s'agit de ceux qui font le mal en secret et le bien en public. On y trouve la même succession: d'abord la référence aux sépulcres blanchis puis la citation de Mt 6,1. Le canon 9 s'achève par une interrogation: "à quoi sert de faire semblant de jeûner et d'être abstinent devant les hommes, si dans nos lieux secrets nous engloutissons un bœuf ou un cheval?" qui ramène à la question alimentaire. De même dans le chap. 3, 35, à la fin, Grégoire rappelle les conseils de Paul aux Corinthiens: "Paul disait à des disciples qui prenaient des aliments impurs, sans contracter certes de souillure, mais en étant par là pour des frères encore imparfaits une cause de tentation..."

Dans le canon 10 on trouve d'abord des prescriptions très concrètes: le prêtre ne doit pas célébrer l'eucharistie les jambes nues, il faut consacrer du pain et non de la croûte, le calice ne doit pas être en corne de bœuf. Ensuite il s'agit d'interdire aux évêques de juger des affaires séculières avec l'argument de II Tim 2, 4, "Personne combattant pour Dieu ne s'implique dans les affaires séculières, pour qu'il combatte pour celui pour lequel il s'est entraîné". Cette même citation se trouve dans le chap. 2, 7 du Pastoral avec un commentaire sur la nécessité d'établir des suppléants pour servir les intérêts temporels des peuples. Catherine Cubitt a montré que les canons de ces conciles anglais de 786 portent la marque de l'influence d'Alcuin peut-être par l'intermédiaire de "fiches" remises au légat Georges d'Ostie. Elle a montré les liens avec certaines lettres d'Alcuin ainsi qu'avec certains traités tel que le De virtutibus et surtout l'Admonitio generalis de 789. Le fait que Dümmler ait édité cette lettre de Georges d'Ostie dans l'épistolaire d'Alcuin va bien sûr dans le même sens. Il reste qu'on ne sait presque rien de ce qu'écrivait Alcuin avant 786. Cependant la Règle 
pastorale de Grégoire le Grand est très bien connue d'Alcuin et il la recommande chaudement à plusieurs de ses correspondants. C'était évidemment un texte qu'il connaissait de longue date et la présence de ce traité à l'arrière-plan de ces canons doit sans doute beaucoup à Alcuin (13).

La deuxième partie des canons s'occupe des laïcs. On peut encore y voir l'influence du Pastoral mais d'une autre manière. Rappelons que la troisième partie du Pastoral est une sorte de catalogue des catégories de chrétiens selon des états de vie, des situations sociales, des attitudes psychologiques ou morales. Ici les dix canons du deuxième groupe touchent également différentes catégories sociales. Ainsi le canon 11 concerne les rois et les princes, le canon 12 l'ordination des rois, le canon 13 les puissants et les riches, etc... De fait on peut établir une correspondance entre certains canons et certains chapitres du Pastoral. Le canon 12 évoque les figures de Saul, David et Salomon à propos de l'ordination des rois. Dans Past. 3, 4, à propos des inférieurs et des supérieurs, Grégoire évoque I Sam 24: David est en mesure de tuer Saul mais il ne le fait pas, il se contente de couper un bord de son manteau car il ne devait pas étendre la main sur l'oint du Seigneur (christus Domini), c'est la formule qu'on retrouve dans ce canon 12 et qui d'ailleurs soulève beaucoup de questions sur le sacre royal chez les Anglo-Saxons et sur la nature du pouvoir royal à la fin du VIIIè siècle (14). Le canon 13 concerne les puissants et les riches. Ils doivent prononcer des jugements justes mais ils sont aussi prévenus par le prophète, Is. 5, 8-9, "Malheur à vous qui joignez maison à maison et qui ajoutez champ à champ jusqu'aux limites du pays: est-ce que vous ne serez pas seuls à habiter au milieu de la terre?..." Cette même citation se trouve dans Past. 3, 20, un long chapitre sur ceux qui, sensibles aux misères, donnent déjà de leurs biens, et ceux qui tentent 
encore de ravir le bien d'autrui. Le canon 16 concerne le mariage d'une manière générale. On y retrouve la recommandation de I Cor. 7, 2, pour éviter la fornication, que chaque laïc ait son épouse légitime et chaque femme son mari, comme dans Past. 3, 27 qui avertit différemment ceux qui sont engagés dans les liens du mariage et ceux qui sont libres. On pourrait aussi relever le parallèle entre le canon 20 sur la pénitence et les chap. 3, 29 et 3, 30 du Pastoral ainsi que l'homélie 34 sur l'évangile.

Le canon 11 présente un cas intéressant de recomposition de plusieurs éléments grégoriens. Les rois doivent gouverner avec justice mais ils doivent obéir aux évêques. Déjà le Pastoral rappelait de qui dépend tout pouvoir royal en citant Osée 8, 4, "Eux ont régné, mais non pas à cause de moi; des princes se sont levés et je ne les ai pas reconnus". Cette citation revient dans le canon 11. Auparavant ce canon cite Malachie 2, 7, "Les lèvres du prêtre gardent la science et recherchent la loi de sa bouche, parce qu'il est l'ange des armées du Seigneur" pour définir ainsi les prêtres ou les évêques comme des anges qui ne peuvent être jugés par des rois. La même citation se trouve dans Past. 2, 4. Toutefois dans ce chapitre il s'agit d'insister sur la prédication. En effet c'est peut-être en dehors du Pastoral dans d'autres textes grégoriens que des rapprochements apparaissent mieux. Il faut remarquer au milieu du canon 11 la citation de Hebr 13, 17, “Obéissez à vos prélats comme au Seigneur, en effet ils veillent pour vous, car ils rendront compte pour vos âmes". Elle ne se trouve pas dans le Pastoral. En revanche, elle figure en bonne place dans les Moralia in Job, dans le livre 34. Ce livre est consacré au commentaire du Léviathan représentant du pouvoir temporel et de l'antéchrist avec une forte perspective eschatologique.

Le paragraphe 53 tourne autour de cette citation de Hebr 13, 17: "Parce que nous avons dit que les âmes humaines sont tentés d'une part par les affaires charnelles, d'autre part 
par les affaires spirituelles, que ceux-ci écoutent: Toute chair est du foin et sa gloire la fleur du foin (Is 40,6). Que ceux-là écoutent ce qu'on dit à certains après des miracles: Vous je ne sais pas qui vous êtes, éloignez-vous de moi, vous tous ouvriers d'iniquité (Lc 13, 27). Que ceux-ci écoutent: Si les richesses affluent, n’y attachez pas le cœur (ps 61, 11). Que ceux-là écoutent que les vierges folles qui venaient avec des fioles vides sont exclues des noces à l'intérieur (Mt 25, 12). A nouveau, parce que nous avons dit que les prélats (supérieurs) sont tentés d'une manière et les sujets (inférieurs) d'une autre, que ceux-ci écoutent ce qui est dit par un sage: Ils t'ont établi chef? Ne te dresse pas, mais sois parmi eux comme l'un d'entre eux (Sir 32, 1). Que ceux-là écoutent: Obéissez à vos prélats comme au Seigneur, en effet ils veillent pour vous, car ils rendront compte pour vos âmes (Hebr 13, 17). Que ceux-ci écoutent, lorsqu'ils se glorifient du pouvoir reçu, ce qu'on dit au riche qui brûle par la voix d'Abraham: Souviens-toi, fils, que tu as reçu les biens dans ta vie (Lc 16, 25). Que ceux-là écoutent, lorsqu'ils se dressent en querelles contre leurs recteurs, ce qu'on répond par les voix de Moïse et Aaron au peuple qui murmure: Et votre murmure n'est pas contre nous, mais contre le Seigneur. Nous en effet que sommes-nous? (Ex 16, 8). Que ceux-ci écoutent: Ils seront troublés devant son regard de père des orphelins et pour les jugements des veuves (ps 67, 5). Que ceux-là écoutent ce qui est dit contre l'orgueil des sujets: Celui qui résiste au pouvoir, résiste à l'ordre de Dieu (Rom 13,2). En même temps qu'ils écoutent tous: Dieu résiste aux superbes, mais aux humbles il donne la grâce $(\mathrm{Jac} 4,6)$. Que tous écoutent: Il est immonde auprès de Dieu celui qui exalte son propre cœur (Prov 16, 5). Que tous écoutent: Quoi pour les superbes, la terre et la cendre (Sir 10,9)? Contre la peste de cette maladie que nous écoutions tous ce que la Vérité maîtresse enseigne en disant: Eloignez-vous de moi, parce que je suis doux et humble de cœur (Mt 11, 29) (15). 
Tout ce chapitre est construit comme un florilège scripturaire. On y trouve déjà la structure de la Règle pastorale par le balancement entre les uns et les autres; entre ceux qu'on avertit d'une manière et ceux qu'on avertit de la manière opposée. On voit bien que ce chapitre est centré sur la citation de Hebr 13, 17, obéissez à vos prélats. C'est bien sûr la même leçon que le florilège du canon 11 cherche à dispenser auprès des rois et princes de l'Angleterre. Le texte des Moralia était évidemment facilement disponible aussi bien pour Georges d'Ostie que pour Alcuin. Dans le même livre des Moralia on trouve aussi la citation de Malachie sur les lèvres du prêtre (Mor 34, 7, 14). Mais cette citation était chère à Grégoire. Il l'utilisa aussi dans une lettre à l'empereur Maurice en juin 595 (ep. 5, 36). Grégoire protestait avec véhémence contre l'attitude de l'empereur qui l'avait qualifié de "sot" fatuus. Il rappelle la gravité de la situation militaire de l'Italie envahie par les Lombards et il rappelle que les évêques ne peuvent être jugés par le pouvoir temporel; ils sont appelés tantôt dieux, tantôt anges. Il cite alors labia sacerdotis pour montrer que les évêques sont des anges et pour insister à nouveau ensuite sur le fait qu'ils ne peuvent être jugés par le pouvoir temporel. Il s'appuie sur l'Histoire ecclésiastique qui lui fournit l'exemple de Constantin qui refusa de juger les évêques (16). Tout ce passage est directement en rapport avec le canon 11 et pose à nouveau la question de la connaissance de l'épistolaire de Grégoire. Il est tout à fait possible que Georges d'Ostie, averti par Hadrien Ier, ait pu avoir connaissance d'une telle lettre qui donnait à la citation de Malachie un sens parfaitement adapté aux circonstances des conciles anglais.

Il est clair, bien sûr, que tout ne peut être rapporté à l'influence de Grégoire dans les dispositions de ces conciles de 786. En particulier les prescriptions pratiques, comme on l'a 
vu ci-dessus pour la célébration de l'eucharistie, relèvent de traditions canoniques. Parmi les dispositions du canon 19 on trouve des aperçus curieux sur des pratiques peut-être païennes de l'Angleterre saxonne, à moins qu'il ne s'agisse de survivances celtiques. Ainsi ce canon condamne des coutumes dans le marquage des chevaux; on condamne le fait d'inciser les naseaux, de couper la queue, ou encore de réunir les oreilles. S'agissait-il de marquage pour la propriété ou bien de préparation pour des fêtes? Le canon semble indiquer que ces coutumes sont générales et obligatoires sous peine d'exclusion sociale. Un peu plus loin, mais pas immédiatement après, ce canon recommande, sans l'interdire explicitement, d'éviter de manger la viande de cheval, car les Chrétiens orientaux ne le font pas. Il est possible que ces dispositions trahissent des pratiques (païennes) de sacrifice de chevaux et de consommation rituelle (17). On observera simplement que cette recommandation peut être aussi un écho du canon 9 dans la première partie (cf. supra). Le mal en secret c'était avaler un bœuf ou un cheval, dans un contexte proche du Pastoral. Grégoire fournit par ailleurs, dans un long chapitre des Moralia, toutes les significations morales et spirituelles possibles, en bien et en mal, du cheval (18). Un écho du canon 9 dans le canon 19 ou inversement suppose une conscience nette d'une organisation des canons en deux parties, l'une pour les clercs l'autre pour les laïcs et c'est bien ce qui a déjà été attribué à l'influence d'Alcuin.

Dans ce même canon 19 on en trouve un autre cas: la condamnation d'une pratique de parure humaine: "Dieu en effet a formé un homme beau dans son aspect et son visage, mais les païens l'ont recouvert par l'instinct diabolique de cicatrices monstrueuses, comme dit Prudence: "Il a teint même le sol innocent de taches sordides" (Prudence, Diptychon I, 3). Il semble faire injure au Seigneur celui qui souille et salit sa créature. Certainement si quelqu'un supportait cette injure de la teinture au nom de Dieu, il en recevrait une grande récompense. 
Mais celui qui fait cela à cause de la superstition des païens, n'en tire pas profit pour son salut..." Il s'agit vraisemblablement de pratiques de tatouages. Ces pratiques peuvent être associées aux Celtes. On sait que l'homme du marais de Lindow, dans les environs de Manchester, a été retrouvé dans un état de conservation remarquable à cause de la tourbe. Il est mort vers le début de notre ère (milieu du Ier siècle) et a sans doute été sacrifié. L'état de conservation des tissus organiques a permis de constater la présence de pigments sur la peau. Ces pratiques de peintures ou de tatouages étaient connus des Pictes, ce qui d'ailleurs avait permis aux Romains de donner une étymologie latine à ce nom de peuple (19). Et c'est peutêtre en Northumbrie, comme le suggère Patrick Wormald, que cette pratique se poursuivait encore au VIIIè siècle (20). Dans la formulation de cette condamnation, on peut aussi voir un écho du canon 4 qui concerne les vêtements des moines et des chanoines: “qu'ils vivent dans cet habit dans lequel vivent les moines orientaux et les chanoines à l'exemple des orientaux et non pas avec les couleurs des teintures de l'Inde ou avec un vêtement précieux". La condamnation de vêtements luxueux pour les moines et les ecclésiastiques se retrouvent régulièrement dans les canons conciliaires. Mais on peut s'arrêter sur la forme utilisée ici. Pourquoi ces couleurs des teintures de l'Inde, bien éloignée de l'Angleterre du VIIIè siècle? Le texte latin dit: non tinctis Indie coloribus et veste preciosa. La teinture de l'Inde pour les vêtements monastiques semble ainsi annoncer la teinture des corps laïcs. Or l'expression peut trouver son origine chez Grégoire le Grand.

Il commente ce verset de Job: “[La sagesse] n'est pas comparable aux étoffes teintes de l'Inde” (Job 28, 16). “Que faut-il comprendre par l'Inde d'où vient un peuple noir sinon ce monde dans lequel la vie des hommes est rendue obscure par la faute? Les étoffes teintes de l'Inde sont les sages de ce monde qui, bien qu'enlaidis par l'infidélité et parfois par l'action, 
sont cependant aux yeux des hommes recouverts de la couleur surajoutée de l'honnêteté. Mais la sagesse coéternelle de Dieu n'est pas comparable aux étoffes teintes de l'Inde car tous ceux qui la comprennent en vérité reconnaissent combien elle est éloignée de ces hommes que le monde a honoré comme des sages. Et les paroles mêmes de ses ordres se distinguent des sages de ce monde: quand ceux-ci recherchent l'éloquence leurs paroles prennent comme l'apparence de la beauté par le fard de la teinture, et lorsqu'ils manquent d'une vertu réelle, ils prétendent mensongèrement qu'ils sont autre chose que ce qu'ils sont par l'arrangement des mots comme par des couleurs surajoutées. En revanche la doctrine de la sagesse est belle par la prédication et remarquable par la pure vérité; et elle ne prétend pas de manière fallacieuse être autre chose à l'extérieur, et elle ne garde pas autre chose à l'intérieur; et dans ses paroles elle ne désire pas qu'on la voit belle par l'éclat du discours mais par l'intégrité de la vérité. Donc dans ses ordres la sagesse de Dieu n'est pas comparable aux étoffes teintes de l'Inde; puisqu'elle n'a pas les ornements fardés de l'éloquence, elle plaît comme un vêtement sans teinture. C'est à bon droit que Paul avait méprisé le fard de la teinture lorsqu'il disait: "nous ne disons pas cela dans les mots savants de la sagesse humaine mais dans la doctrine de l'esprit" (I Cor 2, 13). Il préférait en effet montrer cette sagesse dans la seule pureté de la vérité et non pas la recouvrir de fard dans la teinture du discours".

Le verset de Job en question fait partie d'un ensemble sur la sagesse: “On ne donne pas de l'or purifié pour elle et on ne l'échange pas contre de l'argent; elle n'est pas comparée aux couleurs teintes de l'Inde, ni à la pierre de sardoine la plus précieuse, ni au saphir..." (22) Il s'agit du texte de la Vulgate, donc de saint Jérôme, car dans le texte hébreu comme dans la Septante, entrent comme éléments d'évaluation de la sagesse les métaux précieux et les pierreries, mais non les étoffes peintes de l'Inde (23). Ainsi le texte de Jérôme permettait à 
Grégoire de commenter cette expression des étoffes teintes de l'Inde et en assurait aussi la diffusion. Grégoire lui-même insiste à nouveau sur cette expression dans un second passage des Moralia. L'expression se retrouve aussi dans l'abrégé de Lathcen (24).

On peut conclure en considérant cette lettre-rapport de Georges d'Ostie comme un "laboratoire" des pratiques carolingiennes, comme un précurseur de la législation carolingienne, en particulier 1'Admonitio generalis. Certes les aspects proprement anglosaxons sont bien présents: la présence des rois de Mercie et de Northumbrie auxquels s'ajoutent le roi du Wessex en Mercie et dans les deux synodes plusieurs grands laïcs; la présence des grands ecclésiastiques, l'archevêque de Canterbury, l'archevêque de York et beaucoup d'autres évêques y compris un évêque irlandais; certaines pratiques condamnées par les conciles se rapportent également à la société de l'Angleterre saxonne et celtique. Mais les légats romains se présentent non seulement comme les envoyés de Rome mais aussi, au moins indirectement, comme des envoyés du roi franc. Ils sont accompagnés de Wigbod qui est, très officiellement, envoyé de Charlemagne. Enfin les deux lectores, Alcuin et Pyttel, ont aussi un lien avec le monde franc. Dans le cas de Pyttel, nous savons seulement qu'il accompagna Liudger en Frise. En revanche Alcuin est déjà à cette date au service de Charlemagne. Les conciles anglais se déroulent à un moment où il se trouve lui-même en Northumbrie. Sur sa personne se trouvent reliés les projets de l'Eglise anglaise, de l'Eglise romaine, des royaumes anglo-saxons et surtout sans doute du royaume franc et de l'Eglise franque. On notera sur ce point la nécessité de proclamer les canons du concile tant en latin qu'en theodisca ce qui annonce évidemment le concile de Tours de 813. Grégoire le Grand n'est certes pas le seul inspirateur des réformes religieuses et politiques de la fin du VIIIè siècle, mais il faut rappeler 
son importance pour les Anglais, pour Bède, pour Alcuin et à la fin du VIIIè siècle la conjonction d'intérêts autour de l'œuvre du grand pape. Or parmi les œuvres de Grégoire, la Règle pastorale était l'une des plus facilement accessibles, en tout cas bien connue d'Alcuin ou de Boniface avant lui. On peut rappeler que le seul manuscrit qui conserve les canons de Cloveshoe de 747, le BL Cotton ms Otho A. i., de la fin du VIIIè siècle, contient aussi des extraits du Pastoral ainsi qu'une lettre de Boniface à l'archevêque de Canterbury. L'influence du Pastoral, dans les canons de 786, peut être médiatisée par d'autres textes, en particulier les Sentences d'Isidore mais la conscience du rattachement à Grégoire à la fois en l'invoquant et en utilisant ses textes reste bien réelle.

Notes

1 Cf. Alcuini epistolae, E. DÜMMLER ed., MGH epistolae IV, 1895, ep. 3, p. 19-29. Le seul manuscrit contenant les canons de 786 est le codex Guelferbytanus (Wolfenbüttel), Helmstad. 454, du Xè siècle, sur les folios $113 \mathrm{v}$ à $127 \mathrm{v}$, provenant de Saint-Maximin de Trèves. HEFELE-LECLERCQ, Histoire des conciles, III-2, Paris 1910. F.M. STENTON, AngloSaxon England, Oxford 2ème ed. 1947, p. 214-217. Margaret DEANESLY, The PreConquest Church in England, Londres 1961, p. 223-226. Hanna VOLLRATH, Die Synoden Englands bis 1066, Konziliengeschichte, Paderborn München 1985, en part. p. 162-181, rappelle que le synode mercien de 786 avait jusque là attiré l'attention essentiellement à cause de la question de l'érection de Lichfield en archevêché, or cette question-là n'apparaît pas du tout dans le rapport de Georges d'Ostie. Patrick WORMALD, In search of Offa's lawcode, 
dans Ian WOOD and Niels LUND eds, People and Places in Northern Europe 500-1600. Essays in honour of Peter Hayes Sawyer, Woodbridge 1991, p. 25-46. Catherine CUBITT, Anglo-Saxon Church Councils c. 650 - c. 850, Leicester University Press 1995, p. 153-190 et p. 270-271. Donald A. BULLOUGH, Alcuin: Achievement and Reputation, Leiden/Boston, Brill (Education and Society in the Middle Ages and Renaissance 16) 2004.

2 Les canons sont précédés d'un titre situant le synode in Anglorum Saxonia au temps du pape Hadrien Ier et du roi des Francs Charles avec la date (18ème année du règne, 786, indiction 10, et les noms des légats romains: Georges évêque d'Ostie et Théophylacte évêque de Todi. Un préambule explique que les légats ont d'abord participé à un concile avec le roi de Mercie Offa et le roi du Wessex Cynewulf (qui fut d'ailleurs tué un peu plus tard en 786). Georges d'Ostie précise ensuite que Théophylacte est allé dans le royaume de Mercie (apparemment ils s'y trouvaient déjà) et dans les régions de la Bretagne (sans doute à l'ouest ou au sud-ouest de la Mercie); lui-même s'est dirigé vers la Northumbrie en compagnie de l'abbé Wigbod envoyé de Charlemagne (Georges d'Ostie était aussi évêque d'Amiens) et ils ont participé à un concile avec le roi de Northumbrie et l'archevêque d'York.

3 Eanbald archevêque d'York 780-796; Aelfwald roi de Northumbrie 779-788; Tilberthus évêque de Hexham 781-789, Hygbaldus évêque de Lindisfarne 780-803, Aedilberthus évêque de Candida-Casa 777-789, Alduulfus évêque de Mayo (Irlande) consacré par Eanbald d'York en 786, Aethiluuinus évêque d'un siège inconnu; le patricius Sigha qui est en 788 le meurtrier du roi Aelfwald, les ducs Alrich et Siguulfus, Aldberth abbé de Ripon 786-787 et Eghard abbé d'un monastère inconnu. 
4 Ianbert archevêque de Canterbury 766-790; Offa roi de Mercie 757-796; Hugibrethus évêque de Lichfield 779-802, Ceoluulfus évêque de Lindsey 767-796, Unuuona évêque de Leicester (Legor) 781-802, Alchardus évêque de Elmham 786-811, Eadberhtus évêque de Londres, Chunibrectus évêque de Winchester, Harchelus (Heardraedus) évêque de Dunwich, Aeine (Aesne) évêque de Hereford, Tota évêque de Selsey, Waremundus évêque de Rochester, Adalmondus (Aethelmodus) évêque de Sherborne 778-793, Adoredus (Hathoredus) évêque des Hwicce (Wigorn, Worcester), les abbés Edihardus, Alcmundus, Boduuinus, Utel, les ducs Brorda, Eatbaldus, Bertoaldus et le comte Otbaldus.

5 Le préambule de Cloveshoe indique: Praesules... ad seipsos uerba mutuae exhortationis uerterunt, ac sese suumque officium... in omeliis quoque beati patris Gregorii, et sanctorum patrum canonicis decretis, quasi in specula splendiflua considerantes. A.W. HADDAN and W. STUBBS, Councils and Ecclesiastical Documents relating to Great Britain and Ireland, vol. III, Oxford 1871, p. 362-363. cf. H. VOLLRATH, op. cit. p. 150 s.; C. CUBITT, op. cit., p. 99-152. Sur le culte de saint Grégoire, cf. Pierre JOUNEL, Le culte de saint Grégoire le Grand, dans Grégoire le Grand, actes publiés par J. FONTAINE, R. GILLET et S. PELLISTRANDI, CNRS Paris 1986, p. 671-680, Alan THACKER, Memorializing Gregory the Great: the origin and transmission of a papal cult in the seventh and eighth centuries, dans Early Medieval Europe 7, 1998, p. 59-84 et ID. In Search of Saints: The English Church and the Cult of Roman Apostles and Martyrs in the Seventh and Eighth Centuries, dans Early Medieval Rome and the Christian West. Essays in Honour of Donald A. Bullough, Brill Leiden 2000, p. 247-277. 
6 Rappelons que, à la demande de Charlemagne, Hadrien Ier lui envoie le sacramentaire grégorien muni d'une préface qui souligne l'autorité "grégorienne" du texte. Hadrien a également publié la plus grande collection de lettres de Grégoire le Grand dans le Registrum Hadrianum, cf. B. JUDIC, La tradition de Grégoire le Grand dans l'idéologie carolingienne, dans R. LE JAN ed., La royauté et les élites dans l'Europe carolingienne, Lille 1998, p. 1757.

7 ep. 6, 50a MGH = 6, 53 CC; cf. aussi Bède, H.E. I, 23.

8 Selon une indication de Symeon de Durham, Alduulfus a été consacré évêque de Mayo par Eanbald d'York en 786 in monasterio quod dicitur Et-Corabrige c'est à dire Corbridge sur le mur d'Hadrien, qui pourrait bien être aussi le lieu de réunion du synode avec Georges d'Ostie, cf. ed. DÜMMLER p. 28 note 1.

9 C'est la première occurrence de cette expression, cf. H. VOLLRATH, op. cit. p. 172-174 qui l'associe au rôle de Georges d'Ostie et au contexte carolingien. Notons qu'on la retrouve au concile de Tours de 813. Plus généralement cf. Michel BANNIARD, Genèse culturelle de l'Europe, Paris 1989 ; ID., Viva voce. Communication écrite et communication orale du IVè au IXè siècle en Occident latin, Paris Institut des Etudes Augustiniennes 1992.

10 Hanna VOLLRATH, op. cit. p. 166 note 126, suppose qu'ils n'avaient pas une bonne connaissance des relations de Rome avec l'Angleterre car ils semblent ignorer le voyage de 
Théodore et Hadrien en 668-669, mais il s'agit peut-être de se placer plus près de Grégoire lui-même.

11 AUG. Enarr. in ps. 99, par. 2 et sermo 46.

12 Cf. GREGOIRE LE GRAND, Règle pastorale (désigné parfois aussi comme le Pastoral ou encore Liber regulae pastoralis, ou encore liber curae pastoralis) SC 381 et 382, Paris 1992.

13 cf. C. CUBITT, op. cit., p. 180 et B. JUDIC, Introd. à Règle pastorale SC 381, Paris 1992.

14 Ce point du rapport de George d'Ostie est spécialement étudié dans Hanna VOLLRATHREICHELT, Königsgedanke und Königtum bei den Angelsachsen bis zur Mitte des 9. Jahrhunderts, Kölner Historische Abhandlungen 19, Köln Wien 1971 et dans Patrick WORMALD, op. cit. p. 32-33 qui renvoie en particulier à W. LEVISON, England and the Continent in the Eighth Century, Oxford 1946, p. 118-119.

15 Mor. 34, 23, 53, PL 76, col. 747-748.

16 Sur cette lettre voir Marc REYDELLET, La royauté dans la littérature latine de Sidoine Apollinaire à Isidore de Séville, Rome 1981, p. 447 s.

17 Le sacrifice du cheval semble attesté par la sépulture de Childéric à Tournai: Stéphane LEBECQ, Les origines franques, Paris 1990, p. 10-11; sur l'asvamedha, le sacrifice du cheval 
dans l'Inde védique, Mircea ELIADE, Histoire des croyances et des idées religieuses I, Paris 1984. Sur la consommation de la viande de cheval: Bruno LAURIOUX, Manger au Moyen Âge, Hachette 2002. ID. Le lièvre lubrique... dans Anthropozoologica 1988, p. 127-132. Massimo MONTANARI, Alimentazione e cultura nel medioevo, 1989, p. 41, renvoie à Isidore de Séville Etym. 12, 1, 43, à des pénitentiels, au code de Rothari, aux statuts de Corbie. L. G. MUZZARELLI, Norme di comportamento alimentare nei penitenziali, dans Quaderni Medievali 13, 1982. Lynn WHITE, Food and History, dans D.M. WASHER, Food, Man and Society, New York 1976. François SIGAUT, La viande de cheval a-t-elle été interdite par 1'Eglise? dans Ethnozootechnie 50, 1992, p. 85-92. Pierre BONNASSIE, Consommation d'aliments immondes... dans Les sociétés de l'an mil. Un monde entre deux âges, Bruxelles 2001, p. 143-169 souligne le rôle de saint Boniface. Sur l'usage domestique du cheval: Wolfgang Christian SCHNEIDER, Animal laborans : das Arbeitstier und sein Einsatz in Transport und Verkehr der Spätantike und des frühen Mittelalters dans L'Uomo di fronte a l'animale nel'alto medioevo, Settimana di studio del centro italiano di studi sull'alto medioevo 31, Spolète 1985, p. 457-578. Tout récemment : Le cheval au Moyen Âge, sous la direction de Elisabeth Lorans, Tours, PUFR, 2017.

18 Mor 31, 24, 43, PL 76, col. 596-597.

19 Cf. I.M. STEAD, J.B. BOURKE, D.R. BROTHWELL, Lindow Man: the body in the bog, British Museum 1986: Il s'agit de la découverte appelée ensuite Lindow II voir infra, p. 41 les tests pour retrouver des teintures corporelles ont donné des résultats négatifs, p. 162-169 (Ann ROSS) l'analyse des restes alimentaires et des modes de mise à mort rejoint le contexte des 
anciennes cultures celtiques. D.R. BROTHWELL, The bog man and the archaeology of people, British Museum 1986. R. TURNER, R. SCALE eds, Bog bodies. New discoveries and new perspectives, British Museum Press 1995. R. TURNER, Dating the Lindow Moss and other British bog bodies and the problem of assigning their cultural context, dans B. COLES, J.M. COLES, M. SCHOU JØRGENSEN, Bog bodies, sacred sites ans wetland archaeology, Wetland Archaeology Research Project, Exeter 1999, p. 227-234, en part. p. 229: [sur le corps appelé Lindow III découvert en 1987 et datable au radiocarbone entre 30 et 225 de notre ère] "des concentrations inhabituelles de minéraux trouvés dans sa peau ont été provisoirement identifiées comme une peinture corporelle minérale bleue à base de cuivre". Michel PASTOUREAU, Jésus chez le teinturier. Couleurs et teintures dans l'Occident médiéval. Le léopard d'or 1997, l'indigo (lat. indicum, grec indikon = de l'Inde) est issu d'un arbuste indigotier qui pousse en Inde et au Moyen Orient (et en Afrique) mais pas en Europe où il est inconnu au Moyen Age; du coup la couleur indigo parvenant en poudre ou en bloc de poudre compactée est considérée comme une pierre, coûte très cher et cependant présente peu d'intérêt, pas de bleu dans les couleurs liturgiques. Les teintures corporelles chez les anciens Celtes sont mentionnées par César, Guerre des Gaules V, 14, 2 (couleur bleue caeruleum) et Pline, Hist. Nat. XXII, 2, 1 (femmes peintes en bleu foncé).

20 Cf. Patrick WORMALD, op. cit., p. 33.

21 Moralia 18, 46, 74, PL 76 col. 81-82.

22 Job: 28, 15: Non dabitur aurum obryzum pro ea, nec appendetur argentum in 
commutatione ejus; 28, 16: Non conferetur tinctis Indiae coloribus, nec lapidi sardonycho pretiosissimo, vel sapphiro; 28, 17: Non adaequabitur ei aurum vel vitrum, nec commutabuntur pro ea vasa auri.

23 C'est donc Jérôme qui a introduit ce passage, peut-être à cause de colorati Indi dans Virgile Géorgiques 4, 293. Cf. Jacques ANDRÉ et Jean FILLIOZAT, L'Inde vue de Rome. Textes latins de l'Antiquité relatifs à l'Inde. Belles-Lettres 1986. Jacques LE GOFF, L'Occident médiéval et l'Océan Indien: un horizon onirique, dans Pour un autre moyen âge: Temps, travail et culture en Occident 18 essais, Paris 1977, p. 280-298 ne signale pas notre texte qui cependant entre parfaitement dans cet "horizon onirique".

24 "Les teintures très pures ne sont pas composées" (Job 28, 19). Ils sont appelés des teintures très pures ceux qui véritablement sont humbles et véritablement saints, ceux qui savent bien qu'ils n'ont pas par eux-mêmes l'apparence des vertus mais qu'ils tiennent cette apparence du don de la grâce qui survient. Ils ne seraient pas teints en effet s'ils avaient eu naturellement la sainteté. Mais ils sont des teintures très pures parce qu'ils gardent humblement la grâce des vertus qu'ils ont reçue et qui survient en eux-mêmes. C'est pourquoi on dit par la voix de l'époux à propos de la sainte Eglise: “Qui est celle-ci qui monte blanchie?" (Cant 6, 9) Parce qu'en effet la sainte Eglise n'a pas naturellement la vie céleste, mais à cause de l'Esprit qui survient, elle est composée de la beauté des dons, on rappelle qu'elle n'est pas blanche mais blanchie. Et il faut noter ceci ci-dessus lorsqu'on disait: “on ne la compare pas aux étoffes teintes de l'Inde", elle n'a pas porté ces étoffes avec des couleurs pures. A cet endroit pour distinguer la teinture des vraies vertus de ce fard des philosophes, en disant les teintures, on a 
ajouté très pures. En effet ils sont correctement nommés des teintures très pures ceux qui d'abord avaient été souillés par des mauvaises œuvres, cependant avec la venue de l'Esprit, ils sont vêtus de la blancheur de la grâce, de sorte qu'ils apparaissent être autrement loin de ce qu'ils étaient. C'est pourquoi même le baptême, c'est à dire une teinture, est dit notre descente dans l'eau. Nous sommes teints certes, et nous qui d'abord étions enlaidis par la difformité des vices, ayant reçu la foi nous sommes rendus beaux par la grâce et l'ornement des vertus. Mor 18, 53, 87, PL 76 col. 91. Lathcen, Ecloga de Moralibus lib. 18. L'expression se trouve encore dans Beatus de Liebana, Adversus Elipandum lib. 1, cap. 57 et dans Rathier de Vérone, Praeloquia lib. 4, tous deux grands lecteurs de Grégoire le Grand. 